\title{
МАРКЕРЫ ДИСФУНКЦИИ ЭНДОТЕЛИЯ В ПРОГНОЗЕ РАЗВИТИЯ ОРГАННОЙ НЕДОСТАТОЧНОСТИ У БОЛЬНЫХ С ТЯЖЁЛЫМИ ФОРМАМИ РОЖИ
}

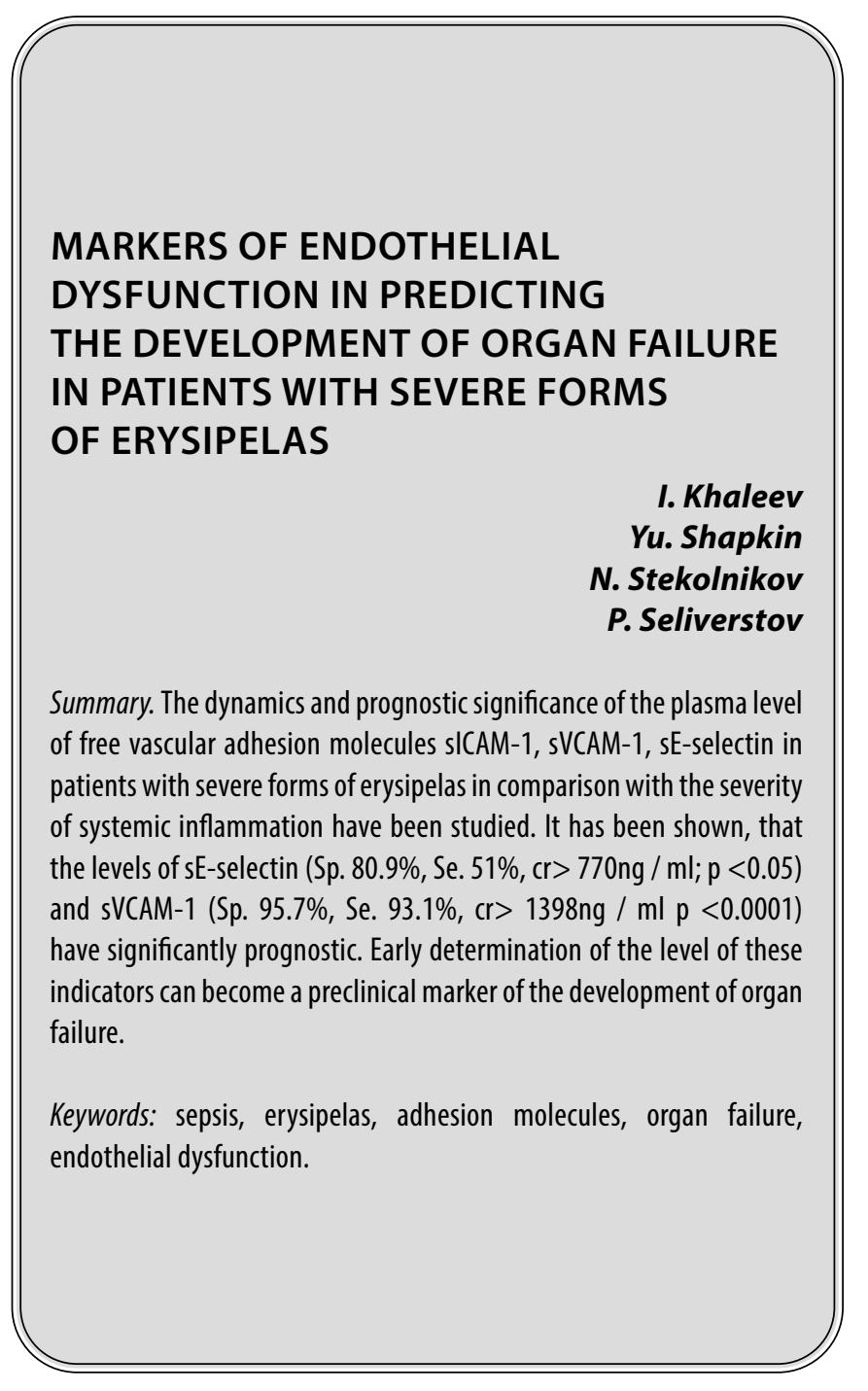

\section{Актуа^ьность}

$\mathbf{5}$ ольные с тяжёлыми формами рожи являются одной из наиболее угрожаемых групп по развитию сепсиса среди пациентов с гнойно-некротическими инфекциями мягких тканей [1]. Изменение диагностических критериев сепсиса с принятием постулатов концепции «Сепсис-3» в 2016 году привело в определённой мере к стандартизации постановки диагноза «сепсис» при регистрации у больного органной недостаточности [2]. Своевременная диагностика синдрома органной и полиорганной недостаточности
Халеев Иван Александрович

Аспирант, ФГБОУ ВО «Саратовский государственный медицинский университет имени В.И. Разумовского» Минздрава России khaleev_64@mail.ru

Шапкин Юрий Григорьевич Д.м.н., профессор, ФГБОУ ВО «Саратовский государственный медицинский университет имени В.И. Разумовского» Минздрава России shapkinyurii@mail.ru

Стекольников Николай Юрьевич

К.м.н., дочент, ФГБОУ ВО «Саратовский государственный медицинский университет имени В.И. Разумовского» Минздрава России nimph2008@yandex.ru

Селиверстов Павел Андреевич

К.м.н., дочент, ФГБОУ ВО «Саратовский государственный медицинский университет имени В.И. Разумовского» Минздрава России seliwerstov.pl@yandex.ru

Аннотация. В работе изучена динамика и прогностичесская значимость плазменного уровня свободных сосудистых молекул адгезии sICAM-1, sVCAM-1, sE-selectin y больных с тяжелыми формами рожи в сопоставлении с тяжестью системного воспаления. Показано, что при поступлении больного прогностическую значимость имеет уровень sE-selectin (Sp. $80,9 \%$, Se. 51\%, сr $>770$ нг/мл; р <0,05) и sVCAM-1 (Sp. 95,7\%, Se. 93,1\%, $\mathrm{cr}>1398$ нг/мл $\mathrm{p}<0,0001)$. Раннее определение уровня данных показателей может стать доклиническим маркером развития органных дисфункций.

Ключевые слова: сепсис, рожа, молекулы адгезии, органная недостаточность, эндотелиальная дисфункция.

становится одномоментно прямым клиническим указанием на развитие у больного сепсиса. При этом каких-либо изменений во взглядах на патогенетические аспекты развития сепсиса не произошло. Синдром системной воспалительной реакции (ССВР) остается ведущим механизмом системной альтерации и формирования органных недостаточностей, в том числе поздних [3]. Как известно, основной «удар» ССВР за счет цитокинов и эндотоксинов получает сосудистый эндотелий. Именно повреждение эндотелия конкретного органа в конечном итоге является ведущим патоморфологическим субстратом, определяющим тяжесть нарушений 
его дисфункции [4]. В настоящее время одним из наиболее чувствительных и достоверных методов оценки эндотелиальной дисфункции является определение уровня сосудистых молекул адгезии $[5,6]$.

Поэтому представляет клинический интерес изучение и оценка динамики плазменного уровня свободных сосудистых молекул адгезии в прогностическом аспекте формирования органных дисфункций у больных с тяжёлыми формами рожи, т.е. оценка прогностической значимости тяжести эндотелиальной дисфункции в развитии хирургического сепсиса у данной категории больных.

\section{Le^ь \\ исслеАОвания}

Изучение прогностической значимости определения тяжести эндотелиальной дисфункции (ЭД) на основании плазменного уровня свободных сосудистых молекул адгезии (ССМА) в ранней диагностике органной недостаточности у больных с тяжёлыми формами рожи нижних конечностей.

\section{Материалы и метомы}

В исследование включены 96 человек: группы исследования больных и группы сопоставления (практически здоровые люди) из 20 человек (10 мужчин и 10 женщин) той же возрастной категории. Изучено клиническое течение стрептококковой инфекции и уровень свободных сосудистых молекул адгезии (sICAM-1, sVCAM-1, sE-selectin) у 76 больных, находившихся на стационарном лечении в клинических базах общей хирургии Саратовского государственного медицинского университета 2015 года по 2020 годы.

Сформированы критерии включения: наличие у больного рожи, или некротизирующей стрептококковой инфекции первого уровня с локализацией на нижних конечностях, наличие у больного признаков системной воспалительной реакции или клинико-лабораторных критериев сепсиса. При этом анализ полученных результатов проводили с учетом подходов, отраженных в концепции «Sepsis-3», 2016.

Подобные больные являются самыми «проблемными», поскольку на течение заболевания оказывает влияние множество факторов, таких как сопутствующая патология и фоновая патология, в первую очередь сахарный диабет и расстройства регионарного кровообращения и лимфооттока.

Сформированы критерии исключения: больные, имеющие стрептококковую инфекцию кожи и мягких тканей отличной локализации, больные старше 80 лет, больные у которых инфекция развилась при наличии злокачественного новообразования, больные с явлениями сердечной недостаточности III стадии и декомпенсированной артериальной недостаточностью нижних конечностей.

Исключение названной категории больных связано с тем, что по данным литературы указанная выше патология изначально характеризуется тяжёлой дисфункцией эндотелия.

Наличие стрептококковой инфекции при эритематозной роже диагностировали клинически, при деструктивных формах проводили забор материала с последующим бактериологическим исследованием.

Оценку эндотелиальной дисфункции проводили путем определения sICAM-1, sVCAM-1, sE-selectin, методом твердотельного иммуноферментного анализа (ELISA) с использованием наборов реактивов (BMS201, BMS232, BMS205) фирмы BenderMedsystems (Австрия). Забор крови осуществляли в следующие сроки: при поступлении; при регистрации утяжеления состояния с развитием органной недостаточности; в ряде случаев диагноз сепсис выставлялся сразу при поступлении, поэтому срок забора крови для определения молекул адгезии совпадал со сроком поступления. Кроме того, у больных, имевших только проявления ССВР, проводили оценку уровня молекул адгезии по купировании явлений системного воспаления. У всех больных с благоприятным исходом оценивали уровень молекул адгезии при выписке.

В группе септических больных сравнительный анализ проводили, ранжировав пациентов по тяжести состояния на основании шкалы SOFA. Необходимо отметить, что все больные, состояние которых оценивалось в 8 и более баллов по шкале SOFA, имели нестабильную гемодинамику и требовали симпатомиметической поддержки, а в 28\% (12 больных) органная дисфункция прогрессировала и регистрировалась клинико-лабораторно уже в стационаре (максимально в течение 4 суток с момента поступления).

Анализ полученных данных проводили в программе Medcalc 11.5. При анализе данных применялись методы непараметрической статистики, в виде медианы, 25го и 75-го перцентилей (Ме [25p;75p]). Сравнительный анализ динамики исследуемых показателей в группе проводили на основе анализа U-критерий Манна-Уитни и дисперсионного анализа повторных измерений. Для оценке прогностически неблагоприятных значений уровня ССМА использовали построение и сравнение ROC-кривых. 
Таблица 1. Динамика ССМА группы сравнения в сопоставлении с тяжестью состояния больных

\begin{tabular}{|c|c|c|c|c|c|c|c|c|c|}
\hline \multirow{3}{*}{$\begin{array}{l}\text { Тяжесть } \\
\text { инфекции, (n) }\end{array}$} & \multicolumn{9}{|c|}{ Показатель Ме [25p;75p], нг/мл } \\
\hline & \multicolumn{3}{|c|}{ sICAM-1, n } & \multicolumn{3}{|c|}{ sVCAM-1, n } & \multicolumn{3}{|c|}{ sE-selectin, $\mathbf{n}$} \\
\hline & 1 & 2 & 3 & 1 & 2 & 3 & 1 & 2 & 3 \\
\hline CCBP, $(45,30,30)$ & $\begin{array}{l}722[690 ; \\
927]\end{array}$ & $\begin{array}{l}604 \\
{[552 ;} \\
842]\end{array}$ & $\begin{array}{l}378^{*} \\
{[340 ;} \\
424]\end{array}$ & $\begin{array}{l}2050 * * \\
{[1946 ;} \\
2320]\end{array}$ & $\begin{array}{l}604 \\
* * \\
{[630 ;} \\
888]\end{array}$ & $\begin{array}{l}402 \\
{[360 ;} \\
530]\end{array}$ & $\begin{array}{l}501 \\
{[450 ;} \\
576]\end{array}$ & $\begin{array}{l}230 \\
{[190 ;} \\
274]\end{array}$ & $\begin{array}{l}78 \\
{[82 ;} \\
112]\end{array}$ \\
\hline $\begin{array}{l}\text { SOFA 2-5 баллов } \\
(15,15,15)\end{array}$ & $\begin{array}{l}1270 \\
* * \\
{[1024 ;} \\
1590] \\
\end{array}$ & $\begin{array}{l}704 \\
{[664 ;} \\
887]\end{array}$ & $\begin{array}{l}540^{*} \\
{[518 ;} \\
612]\end{array}$ & $\begin{array}{l}2600 \\
{[2404 ;} \\
2844]\end{array}$ & $\begin{array}{l}850^{* *} \\
{[630 ;} \\
920]\end{array}$ & $\begin{array}{l}602 \\
{[558 ; 860]}\end{array}$ & $\begin{array}{l}710^{* *} \\
{[634 ;} \\
815]\end{array}$ & $\begin{array}{l}251 \\
{[220 ;} \\
285]\end{array}$ & $\begin{array}{l}100[92 ; \\
124]\end{array}$ \\
\hline $\begin{array}{l}\text { SOFA (6 и более } \\
\text { баллов) }(15,15,15)\end{array}$ & $\begin{array}{l}1554 \\
{[1247 ;} \\
1850]\end{array}$ & $\begin{array}{l}730 \\
{[691 ;} \\
910]\end{array}$ & $\begin{array}{l}575[480 ; \\
628]\end{array}$ & $\begin{array}{l}2680 \\
{[2484 ;} \\
2908]\end{array}$ & $\begin{array}{l}1209[1054 ; \\
1438]^{*}\end{array}$ & $\begin{array}{l}663 \\
{[572 ;} \\
920]\end{array}$ & $\begin{array}{l}790 \\
{[748 ;} \\
965]\end{array}$ & $\begin{array}{l}264 \\
{[242 ;} \\
300]\end{array}$ & $\begin{array}{l}110 \\
{[96 ;} \\
128] \\
* *\end{array}$ \\
\hline (норма), 20 & \multicolumn{3}{|c|}{$302[270 ; 330]$} & \multicolumn{3}{|c|}{$324[290 ; 370]$} & \multicolumn{3}{|c|}{$70[52 ; 90]$} \\
\hline
\end{tabular}

Примечание: 1 - при поступлении больного у всех больных (или регистрации явлений органной в стационаре дисфункции у больных с сепсисом), 2 - при купировании явлений ССВР (или органной дисфункции), 3 - при выписке; ${ }^{*}$ - ( $\left.<<0,05\right)$ различия достоверны в подгруппе по отношению к предшествующему измерению; ** ( $<0,05)$ - различия достоверны по отношению к данным в других подгруппах

\section{Результаты}

Для оценки степени ЭД мы провели ретроспективное ранжирование больных по степени тяжести состояния: CCBP, балами по шкале SOFA. Оценку ЭД в группе сравнения проводили в следующие сроки. На момент поступления регистрировали у всех пациентов (76 человек). Затем по купированию ССВР у всех больных, и по выписке, отражавшей купирование как местного, так и системного воспаления также у всех больных.

Анализ уровня ССМА, представленный данными таблицы, показывает, что у больных с тяжёлыми формами стрептококковой инфекции в момент поступления отмечается значительное повышение плазменного уровня sICAM-1 (от 1,8 до 6 раз превышающее нормальные значения). При этом в группах больных, имевших органную дисфункцию и группе пациентов с ССВР, показатели sICAM-1 также достоверно различаются (выше, примерно, в 1,5 раза у больных с сепсисом, $\mathrm{p}<0,01)$. У больных, не имевших явлений органных дисфункций, при выписке мы не фиксировали достоверных различий уровня sICAM-1 по отношению к условной норме, однако в группе пациентов, имевших органные дисфункции, сохраняется достоверно высокий уровень sICAM-1 при выписке по отношению к нормальным показателям.

Оценивая динамику sVCAM-1, отмечено, что при поступлении у больных отмечается достоверное по отношению к нормальным значениям повышение уровня
ССМА данной категории. Наблюдается восьмикратное повышение уровня sVCAM-1, достоверно не различающееся в группах системного воспалительного ответа и сепсиса. Кроме того, у больных, имевших болеетяжёлые проявления органных дисфункций, после купирования ССВР сохраняется достоверно повышенный уровень sVCAM-1. По стабилизации состояния достоверные различия значений sVCAM-1 регистрируются в группах, имевших явления сосудистой недостаточности и группе септических больных. При этом также сохраняются достоверно высокие значения SVCAM-1 при выписке больных в независимости от тяжести инфекции.

Рассматривая изменения уровня sE-selectin, необходимо отметить, что имеются достоверные отличия содержания sE-selectin в группах с септических больных и больных с ССВР. Также отмечается достоверное, практически семикратное возрастание концентрации данных молекул адгезии по отношению к нормальным показателям. В динамике после купирования явлений системного воспаления достоверных различий в уровне sE-selectin в группах больных не наблюдается, а при выздоровлении уровень sE-selectin не имеет достоверных отличий и соответствует нормальным значениям.

Отдельно нами проведена оценка исследуемых показателей в группе больных, у которых сформировалась поздняя органная дисфункция, что послужило диагностическим критерием сепсиса. Сроки определения ССМА схожи со сроками в вышеописанных подгруппах, однако дополнительно проводили регистра- 
Таблица 2. Динамика ССМА у больных с органными дисфункциями

\begin{tabular}{|c|c|c|c|c|c|c|c|c|c|}
\hline \multirow{3}{*}{$\begin{array}{l}\text { Тяжесть } \\
\text { инфекции, }(n=13)\end{array}$} & \multicolumn{9}{|c|}{ Показатель Ме [25p;75p], нг/мл } \\
\hline & \multicolumn{3}{|c|}{ sICAM-1, день регистрации } & \multicolumn{3}{|c|}{$\begin{array}{l}\text { sVCAM-1, } \\
\text { день регистрации }\end{array}$} & \multicolumn{3}{|c|}{$\begin{array}{l}\text { sE-selectin, } \\
\text { день регистрации }\end{array}$} \\
\hline & 1 & 2 & 3 & 1 & 2 & 3 & 1 & 2 & 3 \\
\hline $\begin{array}{l}\text { поздняя } \\
\text { органная } \\
\text { дисфункция }\end{array}$ & $\begin{array}{l}756 \\
{[560 ;} \\
880]\end{array}$ & $\begin{array}{l}810[760 \\
952]\end{array}$ & $\begin{array}{l}525 \\
{[500 ;} \\
620]^{*}\end{array}$ & $\begin{array}{l}2050 \\
{[1946 ;} \\
2320]\end{array}$ & $\begin{array}{l}1750 \\
{[1440 ;} \\
1950]^{*}\end{array}$ & $\begin{array}{l}620 \\
{[558 ; 840]}\end{array}$ & $\begin{array}{l}500 \\
{[450 ;} \\
610] \\
\end{array}$ & $\begin{array}{l}220 \\
{[200 ;} \\
274]\end{array}$ & $\begin{array}{l}100[89 ; \\
114]^{*}\end{array}$ \\
\hline $\begin{array}{l}\text { условная } \\
\text { норма }\end{array}$ & \multicolumn{3}{|c|}{302 [270;330] } & \multicolumn{3}{|c|}{324 [290;370] } & \multicolumn{3}{|c|}{$70[52 ; 90]$} \\
\hline
\end{tabular}

Примечание - 1,2,3 сроки измерений, описанные выше, * $(p<0,05)$ - различия достоверны в подгруппе по отношению к предыдущему определению;

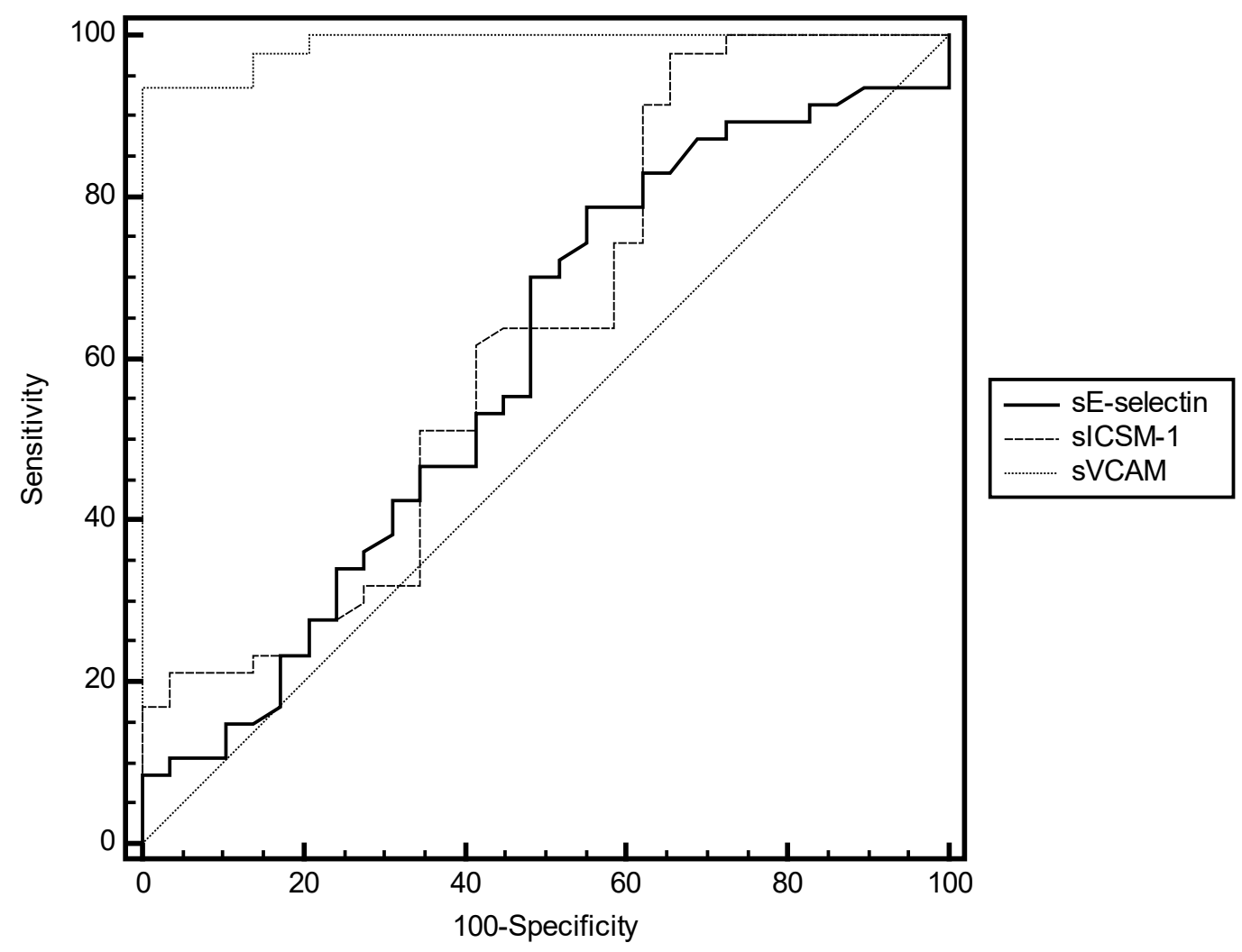

Рис. 1. Сравнительный анализ ROC-кривых по значимости динамики уровня CCMA и формирования органных дисфункций.

цию уровня ССМА в момент фиксации явлений поздней органной дисфункции. Сравнительный анализ данных об уровне ССМА в сроки купирования ССВР в данной ситуации не проводили поскольку при развитии поздних органных дисфункций у пациента могли не регистрировать ССВР, однако лабораторно фиксировали органную дисфункцию.

Результаты определения уровня ССМА 13 больных, у которых ретроспективно зафиксирована поздняя органная недостаточность представлен в таблице 2 .
Из представленных в таблице данных зафиксировано, что достоверных различий содержания ССМА при поступлении не наблюдается, отсутствует достоверные различия в уровне sE-selectin по отношению к больным, имевшим лишь ССВР. Однако по купированию проявлений системного воспаления у больных данной категории отмечается достоверное повышение sICAM-1 и sVCAM-1 по отношению к данным схожей подгруппы. При этом уровень sVCAM-1 остается достоверно высоким и становится предиктором формирования органной дисфункции. 
Таблица 3. Статистические данные сравнительной значимости изучаемых показателей

\begin{tabular}{l|l|}
\hline sE_selectin sICSM_1 & 0,104 \\
\hline Difference between areas & 0,0926 \\
\hline Standard Error ${ }^{c}$ & $-0,0776$ to 0,285 \\
\hline 95\% Confidence Interval & 1,122 \\
\hline Z statistic & $\mathrm{P}=0,2620$ \\
\hline Significance level & 0,322 \\
\hline sE_selectin sVCAM & 0,0659 \\
\hline Difference between areas & 0,193 to 0,452 \\
\hline Standard Error c & 4,891 \\
\hline 95\% Confidence Interval & $\mathrm{P}<0,0001$ \\
\hline Z statistic & \\
\hline Significance level & 0,426 \\
\hline sICSM_1 sVCAM & 0,0679 \\
\hline Difference between areas & 0,293 to 0,559 \\
\hline Standard Error $c$ & 6,276 \\
\hline 95\% Confidence Interval & $\mathrm{P}<0,0001$ \\
\hline Z statistic & \\
\hline Significance level & \\
\hline
\end{tabular}

c Delong et al, 1988(данные программы Medcalc 11.5.0.0)

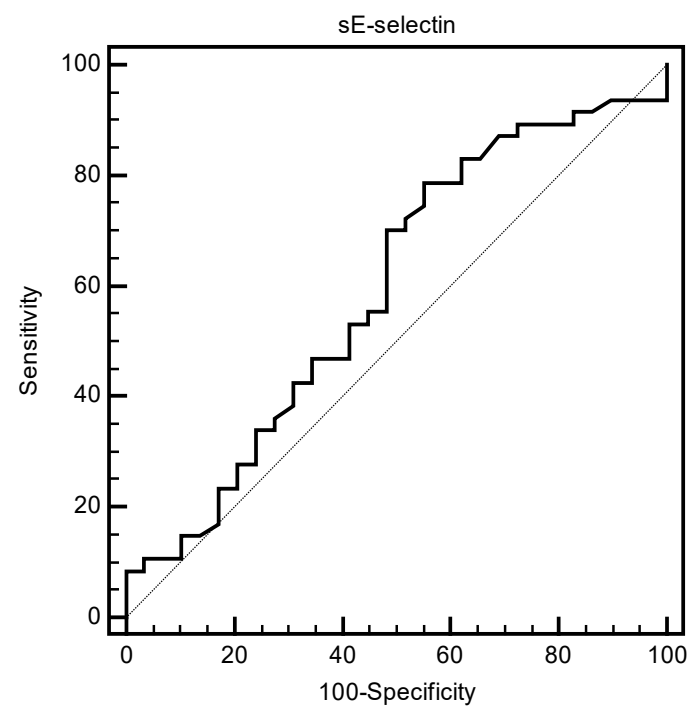

Рис. 2. ROC-анализ значений уровня s-Eselectin в группе сравенения

Для оценки значимости ССМА в диагностике органных дисфункций мы использовали построение ROC-кривых ретроспективно с учетом клинико-лабораторных данных и исхода у каждого больного в конкретный момент времени. Т.е. при поступлении больного или развитии ранних органных дисфункций мы использовали начальные значения ССМА, при формировании поздних органных дисфункций использовали значения на момент клинической регистрации органной недостаточности.
Сравнительный анализ ROC-кривых представлен на диаграмме, (рис. 1).

Статистические данные графика представлены в таблице (табл. 3).

Из данных, представленных на диаграмме и в таблице, видно, что достоверно свидетельствуют о развитии органных дисфункций показатели sE-selectin и sVCAM. 
Таблица 4. Статистические даннык ROC-анализа значимости уровня s-Eselectin

\begin{tabular}{|l|l|}
\hline Area under the ROC curve (AUC) & 0,592 \\
\hline Standard Error ${ }^{a}$ & 0,0658 \\
\hline $95 \%$ Confidence Interval $^{\text {b }}$ & 0,549 to 0,771 \\
\hline z statistic & 2,531 \\
\hline Significance level P (Area=0.5) & 0,0114 \\
\hline
\end{tabular}

c Delong et al, 1988 (данные программы Medcalc 11.5.0.0)

Таблица 5. Статистические даннык ROC-анализа значимости уровня s-VCAM

\begin{tabular}{|l|l|}
\hline Area under the ROC curve (AUC) & 0,989 \\
\hline Standard Error & 0,00765 \\
\hline 95\% Confidence Interval & 0,932 to 1,000 \\
\hline z statistic & 63,923 \\
\hline Significance level P (Area=0.5) & $<0,0001$ \\
\hline
\end{tabular}

Delong et al, 1988(данные программы Medcalc 11.5.0.0)

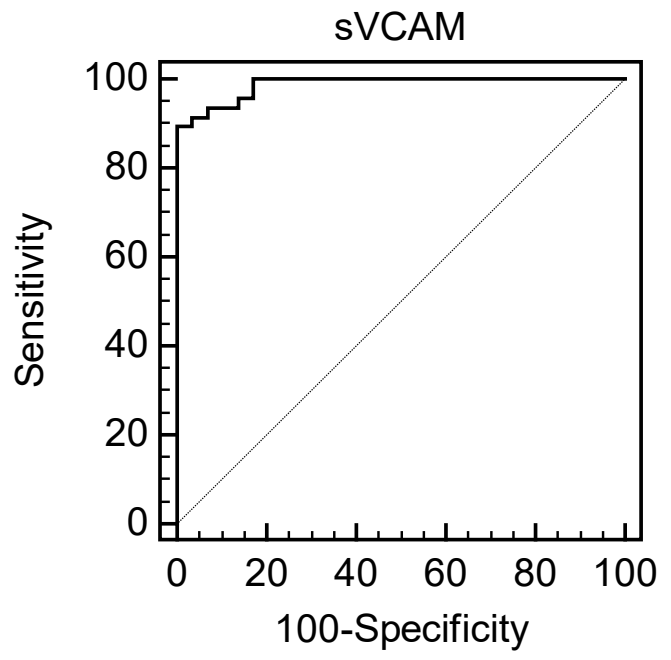

Рис. 3. ROC-анализ значений уровня s-VCAM в группе сравенения

Для расчета пороговых значений показателей провели построение ROC-кривых по каждому показателю в отдельности.

Результаты представлены на диаграммах (рис. 2)

Статистические данные диаграммы представлены в таблице (табл. 4).

Из данных диаграммы, мы видим, что при пороговом значении уровня s-Eselectin до 770 нг/мл достоверно отмечается развитие органных дисфункций, регистрируемых рутинными клинико-лабораторными методами. При этом чувствительность показателя $81 \%$, а специфичность 52\%. На наш взгляд, относительно невысокая чувствительность и низкая специфичность показателя, достоверная при значениях близких к максимальным обусловлена в первую очередь значимостью данного маркера в развитии ранних органных дисфункций, поскольку как описано выше (раздел 3.1) мы не наблюдали достоверных различий данного параметра при оценке развития поздних органных дисфункций.

Несколько иная зависимость регистрируется при анализе уровня SVCAM (рис. 3). 
Статистические данные диаграммы представлены в таблице (табл. 5)

Из полученных данных мы видим, что определение уровня sVCAM является высокочувствительным (95,7\%) и специфичным $(93,1 \%)$ методом как регистрации органных дисфункций, так и предиктором развития поздней органной недостаточности с пороговым значением более 1398 нг/мл.

\section{Обсужление}

Полученные результаты лишь подтверждают то, что ССВР у ряда больных преимущественно оказывает системное воздействие на отдаленные органы с формированием отсроченной органной дисфункции с первичным вовлечением сосудистой стенки в воспалительный ответ. Именно в данной ситуации рутинные клинико-лабораторные исследования до формирова- ния декомпенсации не позволяли прогнозировать формирование органной дисфункции.

\section{Зак^ючение}

Определение уровня дисфункции эндотелия на основании определения динамики свободных сосудистых молекул адгезии является высокоточным прогностическим критерием и предиктором формирования у больного как раннего, так и позднего $\mathrm{CПOH}$, а, следовательно, учитывая наличие инфекционного очага, эффективным методом ранней диагностики сепсиса у больных с тяжёлыми формами рожи.

Динамическая оценка уровня sVCAM является достоверным и высокочувствительным методом прогнозирования сепсиса у данной категории пациентов и может быть использована в оценке эффективности проводимого лечения.

\section{ЛИТЕРАТУРА}

1. Гостищев В.К., Липатов К.В., Комарова Е.А. Стрептококковая инфекция в хирургии // Хирургия. Журнал им. Н.И. Пирогова. 2015. № 12. С. $14-17$.

2. Singer M., Deutschman C.S., Warren S.C, Shankar-Hari M., Djillali A., Bauer M., Bellomo R., Bernard G.R, Chiche J.D, Coopersmith C.M., Hotchkiss R.S, Mitchell M. Levy, Marshall J.C., Greg S. Martin, M. Opal S., Gordon D. Rubenfeld, Van der Poll T., Vincent J.L., Angus D.C. The Third International Consensus definitions for Sepsis and Septic Shock (Sepsis-3). JAMA. 2016. Vol. 315. № 8. P. 801-810.

3. Colbert J.F., Schmidt E.P. Endothelial and microcirculatory function and dysfunction in sepsis. Clin. Chest. Med. 2016. Vol. 37. P. 263-275. D0I: 10.1016/j. ccm.2016.01.009.

4. Jacobi J., The pathophysiology of sepsis - 2021 update: Part 1, immunology and coagulopathy leading to endothelial injury, American Journal of HealthSystem Pharmacy, 2021; zxab380, doi.org/10.1093/ajhp/zxab380.

5. Sousa A., Raposo F., Fonseca S., Valente L., Duarte F., Gonçalves M., Tuna D., Paiva J.A. Measurement of cytokines and adhesion molecules in the first 72 hours after severe trauma: association with severity and outcome. Dis Markers. 2015. P. 747036. DOI: 10.1155/2015/747036.

6. Bui T.M., Wiesolek H.L., Sumagin R. ICAM-1: A master regulator of cellular responses in inflammation, injury resolution, and tumorigenesis. J. Leukoc Biol. 2020. Vol. 108 (3). P. 787-799. DOI: 10.1002/JLB.2MR0220-549R.

( ) Халеев Иван Александрович ( khaleev_64@mail.ru ), Шапкин Юрий Григорьевич ( shapkinyurii@mail.ru ), Стекольников Николай Юрьевич ( nimph2008@yandex.ru ), Селиверстов Павел Андреевич ( seliwerstov.pl@yandex.ru ). Журнал «Современная наука: актуальные проблемы теории и практики» 